\title{
Les parcours de prévention par le sport de l'obésité chez les enfants d'un quartier populaire strasbourgeois : transmissions familiales et rapports à l'activité physique
}

\author{
Marine Grassler ${ }^{1, *}$, Sandrine Knobé ${ }^{2}$, et William Gasparini ${ }^{2}$ \\ ${ }^{1}$ Laboratoire Sport et sciences sociales (E3S; EA 1342), Université de Strasbourg, Le Portique, 14 rue René Descartes, Campus \\ Esplanade, 67084 Strasbourg, France \\ ${ }^{2}$ Université de Strasbourg, E3S EA1342, Strasbourg, France
}

Reçu le 2 mars 2018, Accepté le 20 décembre 2018

\begin{abstract}
Résumé-Depuis 2014, la ville de Strasbourg a mis en place un parcours de prévention intitulé «PRise En Charge Coordonnée des enfants Obèses et en Surpoids de Strasbourg » (PRECCOSS) destiné aux enfants âgés de 3 à 12 ans. Les enfants en situation de surpoids ou d'obésité bénéficient ainsi d'une prise en charge gratuite d'une durée de trois ans avec un suivi individuel et des ateliers collectifs d'activité physique. Dans cette tranche d'âge, la famille joue un rôle important d'accompagnement ou d'incitation mais également de transmission de comportements actifs selon différentes modalités et stratégies, conscientes et inconscientes. L'analyse des transmissions du «goût» à l'activité physique à partir d'une enquête qualitative auprès d'enfants en surpoids et de leurs parents résidant dans un quartier populaire permet d'identifier des logiques familiales différenciées où se conjuguent effet des trajectoires sociales et division sexuée des rôles parentaux.
\end{abstract}

Mots clés : enfant, famille, transmission, activité physique, obésité

\begin{abstract}
The routes prevention through sport for obese children in a popular district of Strasbourg: family transmissions and reports on physical activity. Since 2014, the city of Strasbourg has implemented a prevention program named «PRise En Charge Coordonnée des enfants Obèses et en Surpoids de Strasbourg» (PRECCOSS) for children between 3 and 12 years of age. Children who are overweight or obese can access freely to a specific care during a period of 3 years with individual follow-up and collective physical activity workshops. At this age, the family plays an important role of accompaniment or of incentive but also of transmission either by inculcation (voluntary education), or by impregnation (habitus) of assets parenting behavior. With a qualitative study taking into account overweight or obese children and their parents living in a working-class neighborhood, we analyze transmission of the appetite for physical activity and identify different families' logics, which combine effect of social trajectories and gender division of parenting roles.
\end{abstract}

Keywords: child, family, transmission, physical activity, obesity

\footnotetext{
*Auteur correspondant: mgrassler@unistra.fr
} 


\section{Introduction}

La prévalence du surpoids et de l'obésité, notamment celle des enfants, est aujourd'hui l'une des préoccupations majeures de santé publique en France. La plupart des études montrent que la probabilité qu'un enfant obèse le reste à l'âge adulte varie de 20 à $50 \%$ si elle se développe avant la puberté, et de 50 à $70 \%$ si elle s'installe après la puberté (Whitaker, Wright, Pepe, Seidel, \& Dietz, 1997). L'obésité infantile est également plus fréquente dans les groupes sociaux défavorisés (DREES, 2017 ; Poulain, 2009 ; Saint and Pol (de), 2007, 2008). Ainsi à Strasbourg, les populations les plus touchées par l'obésité vivent dans les quartiers prioritaires de la politique de la ville $(\mathrm{QPV})^{1}$ (Fernandez et al., 2007). Strasbourg est une ville également caractérisée par une forte prévalence du surpoids et de l'obésité chez les enfants. En Alsace, elle dépasse de plus de trois points celle constatée au niveau national $15,3 \%$ en Alsace contre $12,1 \%$ en France métropolitaine pour les enfants âgés de 5-6ans) (Guignon et al., 2010). C'est pourquoi, depuis 2014, a été mis en place un programme de prévention intitulé «PRise En Charge Coordonnée des enfants Obèses et en Surpoids de Strasbourg » (PRECCOSS) destiné aux enfants âgés de 3 à 12 ans. Ce dispositif de prévention a été développé dans le cadre du contrat local de santé $2(\mathrm{CLS} 2)^{2}$ de la ville de Strasbourg $(2015 / 2020)^{3}$. Dans un premier temps, il a été proposé dans trois QPV de Strasbourg où le taux de surpoids et d'obésité des enfants était supérieur à la moyenne de la ville ${ }^{4}$ Élargi depuis

\footnotetext{
${ }^{1}$ Depuis le 21/02/2014, la loi de programmation pour la ville et la cohésion urbaine a défini des Quartiers Prioritaires de la politique de la Ville-QPV (Loi no 2014-173). Ce sont des territoires d'intervention du ministère de la ville. Il y en a environ 1500 en France. C'est le commissariat général à l'égalité des territoires qui identifie et établit les limites de ces quartiers en s'appuyant sur les données de l'Institut national de la statistique et des études économiques (INSEE) concernant le revenu des habitants. Les périmètres des QPV sont fixés par les décrets $\mathrm{n}^{\mathrm{o}} 2014-1750$ (métropole) et $\mathrm{n}^{\mathrm{o}} 2014-1751$ (Outre-mer) du 30/ 12/2014, rectifiés par le décret $n^{\circ} 2015-1138$ du 14/09/2015. Depuis le $01 / 01 / 2015$, ces périmètres se substituent aux zones urbaines sensibles (ZUS) et aux quartiers en contrat urbain de cohésion sociale (CUCS).

${ }^{2}$ Un contrat local de santé est un plan commun d'actions en matière de santé, décidé à l'échelle d'un territoire par deux partenaires : l'Agence Régionale de Santé (ARS), et la commune ou l'intercommunalité.

${ }^{3}$ Il est inscrit dans l'axe 5 du CLS2 (p. 105): «renforcer les actions de nutrition pour diminuer la prévalence du surpoids chez les enfants et lutter contre les maladies chroniques chez les adultes». Cf. Contrat local de santé II de Strasbourg 2015-2020, Ville de Strasbourg, juin 2015, [en ligne] http:/ /www.strasbourg. $\mathrm{eu} /$.

${ }^{4}$ Notamment dans le quartier du Neuhof où la prévalence de l'obésité pour les enfants de 5-6ans en 2009 était de 16,4\% comparativement à 7,4\% pour l'ensemble de l'agglomération strasbourgeoise. Cf. Communauté Urbaine de Santé (2011), Document de diagnostic et d'orientations communautaires.
}

septembre 2016 à tous les quartiers strasbourgeois, il devrait aussi être proposé à moyen terme aux adolescents (13-18 ans).

PRECCOSS offre gratuitement aux enfants inclus dans le programme, une prise en charge avec un suivi individuel et des ateliers collectifs d'activité physique. Entre 2014 et 2015, 169 enfants ont bénéficié de ce dispositif, 60 familles n'ont pas poursuivi une première expérience et 80 familles n'ont pas souhaité y participer (Signorini, Ringenbach, Mutzig, \& Alizada, 2015). S'ils ont été dépistés - par la médecine scolaire ou la médecine de ville, pédiatrique et générale - comme étant en surpoids ou obèses, les enfants scolarisés à Strasbourg sont orientés via une prescription médicale vers le dispositif PRECCOSS. Accompagné de ses parents et muni de son ordonnance, l'enfant est alors incité à prendre rendez-vous avec l'infirmière « référente» du dispositif qui assure son inclusion et son orientation vers les activités et/ou professionnels dédiés à cette prise en charge d'une durée maximale de trois ans ${ }^{5}$.

En réponse à une commande, nous avons construit un projet de recherche ${ }^{6}$ visant à mieux comprendre les raisons de l'engagement - ou du non-engagement-dans une pratique d'activités physiques d'enfants en situation de surcharge pondérale, et dont les parents sont faiblement dotés en ressources économiques. Au cours de l'enfance, la famille joue un rôle important d'accompagnement ou d'incitation aux différentes pratiques de loisirs (Octobre \& Jauneau, 2008) sachant que les modèles éducatifs des familles se différencient socialement (Déchaux, 2009). De nombreuses études ont mis en exergue l'influence que les parents peuvent avoir sur la transmission de «bons comportements » en matière d'activité physique auprès des enfants et des adolescents (Mitchell et al., 2012; O'Connor, Jago, \& Baranowski, 2009 ; Trost, McDonald, \& Cohen, 2013). Cette influence semble s'accentuer lorsque les parents connaissent les recommandations de santé publique et les potentiels bienfaits de l'activité physique, et qu'ils soutiennent leurs enfants dans leur activité (Trost et al., 2013). Dans ce cas, la relation parentenfant pourrait encourager des stratégies de prévention primaire ou secondaire de lutte contre le surpoids et l'obésité infantile ciblant la famille dans son ensemble,

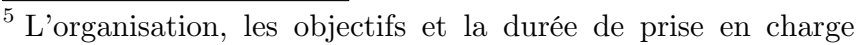
correspondent aux recommandations éditées par la HAS (2011). ${ }^{6}$ Le volet concernant spécifiquement les rapports à l'activité physique constitue un des aspects d'un programme de recherche plus large basé sur une collaboration interdisciplinaire et originale entre deux composantes de formation, le Département de Médecine générale de la Faculté de médecine et la Faculté des sciences du sport de Strasbourg, deux unités de recherche en sciences sociales ( Sociétés, Acteurs et Gouvernance en Europe»-UMR 7363 -, et «Sport et sciences sociales », EA 1342), le service de santé et sport-santé de l'Eurométropole/Ville de Strasbourg, le programme PRECCOSS, une structure, la Maison de Santé du Neuhof et des associations de terrain. Le projet s'intitule «Recherche action sur l'obésité dans les QPV: évaluer les actions publiques et agir ensemble dans les quartiers. Éducation thérapeutique et prévention par l'activité physique».
} 
c'est-à-dire en agissant aussi bien sur l'enfant que sur ses parents (Skouteris et al., 2012). Cependant, de nombreuses études traitent de la nutrition et de l'activité physique chez les enfants sans prendre toujours en compte les critères socio-économiques et/ou le lieu de vie de la famille. Pourtant, "l'obésité est un problème social parce qu'elle touche de façon socialement différenciée les populations » (Poulain, 2009) et renforce les inégalités sociales de santé.

Par conséquent, l'objectif de notre étude consacrée aux enfants en surpoids ou obèses est d'interroger la transmission des parents aux enfants d'un «goût» pour l'activité physique, et ce plus particulièrement dans des familles résidant en quartier populaire. Ainsi, nous cherchons à comprendre de quelle manière ces familles entrent dans le dispositif local PRECCOSS proposé par les professionnels de santé et des activités physiques.

Après avoir explicité l'importance des transmissions familiales dans le rapport des enfants aux activités physiques et précisées la méthodologie mise en œuvre, nous développerons plus en détail leur effet sur les rapports à la santé, à l'activité physique et au surpoids. L'impact des trajectoires et de l'histoire familiale dans la transmission d'habitudes de vie physiques ou sportives sera plus particulièrement étudié, en tenant compte notamment de la division sexuée des rôles parentaux.

\section{Cadre théorique}

Lieu de socialisation primaire (Berger \& Luckmann, 1986; Darmon, 2007), la famille apparaît comme un vecteur fondamental de transmission de pratiques et de valeurs. À ce titre, il importe de saisir comment les parents, notamment, sont susceptibles de transmettre à leurs enfants un goût familialement et socialement construit pour la pratique d'activités physiques, encadrées ou auto-organisées. Analysant les liens d'homologie structurale entre des propriétés sociales et des modes d'engagement physique ou sportif, la construction sociale du goût à l'activité physique a été étudiée par plusieurs sociologues du sport (Defrance, 1995; Pociello, 1999). D'autres travaux mettent également en évidence l'existence de cultures somatiques familiales ${ }^{8}$, de façons différentes de prendre en compte le corps de l'enfant selon les familles, au regard de la position sociale, de la trajectoire familiale, de l'expérience sportive et du rapport

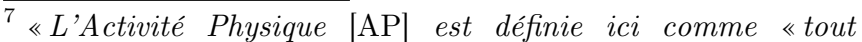
mouvement corporel produit par contraction des muscles squelettiques entraînant une augmentation de la dépense énergétique par rapport à la dépense énergétique de repos» (Caspersen et al., 1985). L'AP regroupe l'ensemble des activités qui peuvent être pratiquées dans différents contextes, en poursuivant des objectifs variés (utilitaires, sanitaires, sociaux, etc.). Les principaux contextes de pratique d'AP sont le travail, les transports, les activités domestiques et les loisirs. Ces derniers incluent l'exercice, le sport et l'AP de loisir non structurée» (ANSES, 2016).

${ }^{8}$ Notamment voir les travaux précurseurs de Boltanski (1971). Les usages sociaux du corps. Annales. Économies, Sociétés, Civilisations, 1, 205-233.
}

à la culture légitime des parents (Neyrand, Mennesson, \& Fine, 2014). La transmission familiale d'un rapport au corps est d'autant plus importante qu'il a déjà été démontré une corrélation entre la situation de surcharge pondérale des parents et celle des enfants. La prévalence est maximale lorsque les deux parents présentent une surcharge pondérale et que l'un des deux est obèse (Vincelet, Galli, \& Gremy, 2006). Les processus de transmission intergénérationnelle sont au cœur de l'analyse sociologique car ils révèlent comment la société se reproduit, se transforme et se perpétue (Attias-Donfut \& Rozenkier, 1995). Au cœur de l'étude des processus éducatifs, les transmissions familiales ont fait l'objet de nombreux travaux, notamment en sociologie du sport, qui montrent comment l'éventuelle pratique sportive des parents influe sur celle de leurs enfants (Defrance, 1995; Mennesson, 2011). Par contre, peu de travaux s'intéressent à ces processus de transmission chez des populations éloignées du monde du sport, par exemple des enfants en surpoids ou obèses. Pourtant, la diffusion de recommandations de santé publique, en matière de pratique régulière d'activités physiques ${ }^{9}$ nous conduit à tenter de comprendre leur réception et leurs effets en termes de changement de comportement (Craggs, Corder, Van Sluijs, \& Griffin, 2011).

Dans ce contexte, parallèlement aux autres acteurs éducatifs (école, programmes de prévention), la famille joue un rôle central: elle peut ou non accompagner et inciter l'enfant à bouger, être actif ou pratiquer un sport par inculcation (éducation volontaire), ou par imprégnation et effet d'imitation (habitus) des comportements actifs parentaux (Octobre \& Jauneau, 2008). Dès lors, l'activité physique est l'une des dimensions du «corps appris » au sein de la famille. Selon Bourdieu (1997: 168), «nous apprenons par corps. L'ordre social s'inscrit dans les corps». Les «techniques du corps » sont éminemment culturelles et, pour une part, transmises au sein du milieu familial (Mauss, 1950). D'autres auteurs ont également montré les processus et les effets d'un apprentissage par corps (Faure, 2000) notamment, pour les membres des classes populaires (Wacquant, 2000). Il en va de même pour ce qui concerne les pratiques vestimentaires, alimentaires ou d'hygiène (Court, Mennesson, Salaméro, \& Zolesio, 2014).

En matière d'éducation à la santé se pose également la question de l'appropriation voire de l'incorporation des comportements recommandés par les campagnes de promotion de la santé par les activités physiques (notamment celles développées dans le cadre des PNNS) (Favier-Ambrosini, 2016; Radel, 2012). En effet, les sociologues du risque (par exemple, Peretti-Watel, 2000)

\footnotetext{
${ }^{9}$ Les principales recommandations sont les suivantes (ANSES, 2016): (1) pour les adultes, pratiquer 30 minutes d'activité physique développant l'aptitude cardio-respiratoire d'intensité modérée à élevée, au moins 5 jours par semaine, en évitant de rester 2 jours consécutifs sans pratiquer; (2) pour les enfants et les adolescents de 6 à 17 ans, pratiquer au moins 60 minutes par jour d'activité physique d'intensité modérée à élevée.
} 


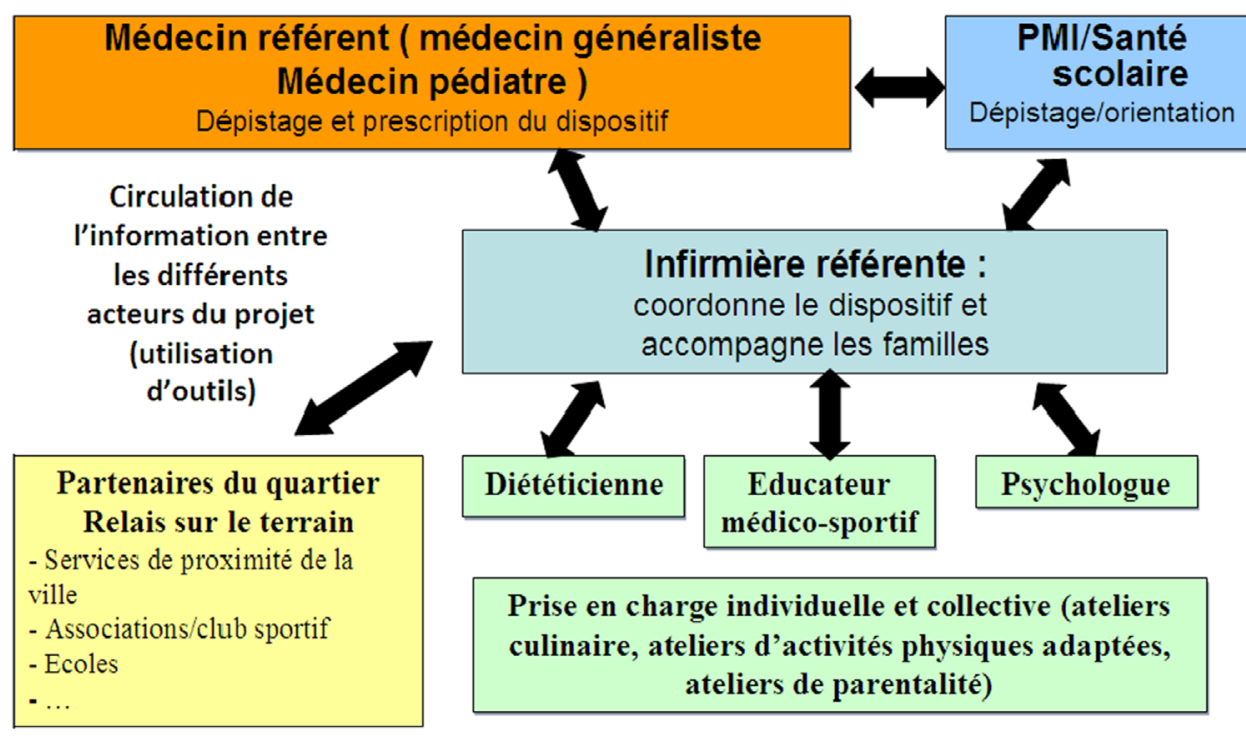

Fig. 1. Représentation schématique du dispositif PRECCOSS : les acteurs de la prise en charge des enfants en surpoids ou obèses. Source: service promotion de la santé et de la personne, ville de Strasbourg.

montrent que les campagnes de prévention touchent davantage certaines catégories sociales 《disposées》 à recevoir les messages. Cette question s'avère d'ailleurs d'autant plus importante que l'on s'intéresse à des populations vulnérables (atteintes de maladies chroniques et/ou précaires) et socialement défavorisées qui ne pratiquent pas nécessairement une activité physique régulière. De nombreuses municipalités ${ }^{10}$ proposent depuis quelques années des dispositifs d'accès et d'incitation à la pratique d'activités physiques destinées principalement à ces populations ${ }^{11}$.

Étudier les transmissions familiales permettrait ainsi de mieux comprendre le rapport des enfants aux recommandations de pratique d'activité physique des politiques de santé publique. L'analyse des discours et pratiques des enfants eux-mêmes, mais également de leur famille (parents et fratrie notamment) permettra d'identifier des logiques familiales différenciées en fonction de facteurs tels que : les représentations des enfants et de leurs parents par rapport à la santé et en particulier au surpoids, l'histoire sociale, migratoire et sportive des parents et l'existence d'une division sexuée des rôles parentaux.

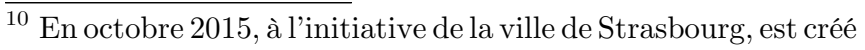
un «réseau national des villes sport santé sur ordonnance», $c f$. http://www.strasbourg.eu/vie-quotidienne/solidarites-sante/ sante/sport-sante-sur-ordonnance-a-strasbourg/le-reseaunational-des-villes-sport-sante-sur-ordonnance.

${ }^{11}$ Des études ont clairement montré que les rapports des bénéficiaires à la pratique sportive et au dispositif de prescription médicale d'activités physiques à Strasbourg sont pluriels et fortement marqués par des logiques sociales d'appréciation des effets de cette pratique et d'incitation des groupes de pairs (Gasparini \& Knobé, 2013; Gasparini, Knobé, \& Didierjean, 2015).
}

\section{Méthodologie}

Le dispositif de prévention de l'obésité par l'activité physique (cf. Fig. 1) permet aux enfants de bénéficier - entre autres - d'un accès gratuit à des séances d'activités physiques hebdomadaires, à des ateliers de cuisine ainsi qu'à un suivi diététique et/ou psychologique. Les ateliers d'activités physiques ${ }^{12}$ sont proposés aux enfants «afin de créer une habitude régulière de pratique physique et sportive, dans un groupe avec d'autres enfants du même quartier, de la même tranche d'âge, ayant la même problématique de surpoids» (Signorini et al., 2016: 33). Tandis que les ateliers culinaires collectifs proposés aux enfants «portent sur la découverte des fruits et légumes par le biais de recettes connues et revisitées» (Signorini et al., 2016: 40) susceptibles d'être facilement reproduites. En fonction des objectifs de l'enfant et de sa famille, formalisés dans un Suivi Personnalisé de Santé (SPS) lors de la première rencontre avec l'équipe pédagogique, la famille pourra bénéficier d'un suivi diététique et/ou psychologique individuel auprès des professionnels du dispositif, en plus des consultations avec l'infirmière coordinatrice qui ont lieu tous les 3 à 6 mois.

Les logiques de transmissions familiales à l'œuvre au sein des familles avec un des enfants en surpoids ou obèse ont été appréhendées grâce à une enquête qualitative. Dix familles ont été interrogées (six participant au dispositif PRECCOSS et quatre n'y participant pas ${ }^{13}$ ) grâce à des entretiens semi-directifs avec l'enfant et au moins l'un de ses parents à son domicile. C'est par l'intermédiaire des

\footnotetext{
${ }^{12}$ Exemples d'activités proposées aux enfants : football, basket, danse, boxe, theque, kinball, uni-hockey, tchoukball, ultimate, flag, badminton, speedball, arts du cirque, grand jeu "totem", acrogym...

${ }^{13}$ Les parents n'ont pas contacté l'équipe PRECCOSS pour un premier rendez-vous.
} 
Tableau 1. Caractéristiques des familles enquêtées.

\begin{tabular}{|c|c|c|c|c|c|c|c|c|}
\hline & Enfant & Sexe & $\begin{array}{l}\hat{\text {AAge }} \\
\text { (années) }\end{array}$ & $\begin{array}{l}\text { Profession } \\
\text { mère }\end{array}$ & $\begin{array}{l}\text { Profession } \\
\text { père }\end{array}$ & $\begin{array}{l}\text { Nombre } \\
\text { de frères } \\
\text { et sœeurs }\end{array}$ & $\begin{array}{l}\text { Pays de } \\
\text { naissance } \\
\text { des parents }\end{array}$ & $\begin{array}{l}\text { Apparence } \\
\text { corporelle } \\
\text { parents }^{\mathrm{a}}\end{array}$ \\
\hline \multirow{6}{*}{$\begin{array}{l}\text { Enfant participant } \\
\text { à PRECCOSS }\end{array}$} & A.R. & $\mathrm{F}$ & 9 & Sans emploi & Sans emploi & 2 & Algérie & $\begin{array}{l}\text { Père et mère, } \\
\text { poids normal }\end{array}$ \\
\hline & $\begin{array}{l}\text { N.S. } \\
\text { N.F. }\end{array}$ & $\begin{array}{l}\mathrm{F} \\
\mathrm{F}\end{array}$ & $\begin{array}{l}9 \\
10\end{array}$ & Sans emploi & Sans emploi & 2 & Maroc & $\begin{array}{l}\text { Mère et père, } \\
\text { situation de surpoids }\end{array}$ \\
\hline & L.L. & $\mathrm{F}$ & 10 & Employée & Ouvrier & 1 & France & $\begin{array}{l}\text { Mère, } \\
\text { poids normal }\end{array}$ \\
\hline & M.D. & $\mathrm{F}$ & 10 & Congé maternité & Ouvrier & 2 & Congo & $\begin{array}{l}\text { Mère, } \\
\text { situation d'obésité }\end{array}$ \\
\hline & K.D. & M & 9 & Sans emploi & Ouvrier & 2 & Russie & $\begin{array}{l}\text { Mère, situation } \\
\text { de surpoids }\end{array}$ \\
\hline & N.Y. & M & 11 & Employée & & 1 & Turquie & Mère, poids normal \\
\hline \multirow{4}{*}{ NON PRECCOSS } & C.M. & $\mathrm{F}$ & 8 & Sans emploi & Ouvrier & 0 & Roumanie & $\begin{array}{l}\text { Mère, situation } \\
\text { de surpoids }\end{array}$ \\
\hline & R.F. & $\mathrm{F}$ & 9 & Sans emploi & Employé & 2 & Maroc & Mère, situation d'obésité \\
\hline & S.M. & M & 9 & Sans emploi & Ouvrier & 3 & Maroc & Mère, situation d'obésité \\
\hline & K.B. & M & 10 & Employée & Employé & 1 & Turquie & $\begin{array}{l}\text { Mère, } \\
\text { poids normal }\end{array}$ \\
\hline
\end{tabular}

a D'après l'appréciation subjective de l'enquêtrice.

professionnels de santé intervenant dans le dispositif PRECCOSS que nous avons été mis en contact avec les familles. Les enfants de l'échantillon avaient été dépistés comme étant en surpoids ou obèses et conviés à participer à PRECCOSS. Pour la plupart immigrée de première génération, les parents, résident depuis plusieurs années dans le QPV du Neuhof ${ }^{14}$ à Strasbourg (cf. Tab. 1). Souvent les mères n'exercent pas d'activité professionnelle et ont, par conséquent, été majoritairement présentes ${ }^{15}$ lors des entretiens avec les enfants.

L'entretien a été rendu plus ludique par quelques aménagements afin de faciliter la parole des enfants qui peut s'avérer difficile à susciter (Auger, Lefrançois, \& Trepied, 2017). Ainsi, pour répondre à la question sur ses activités de loisirs ou ses connaissances en matière de santé, l'enfant devait positionner des gommettes de couleur pour indiquer ses préférences ou connaissances. Les entretiens semi-directifs ont été complétés par un carnet de bord d'activités physiques ${ }^{16}$ distribué à chaque enfant des dix familles enquêtées. L'enfant devait y reporter chaque jour, sur une période de quinze jours, comportant une semaine d'école et une semaine de

\footnotetext{
${ }^{14}$ Un quartier prioritaire de la politique de la ville, avec une part importante de jeunes puisqu'un quart des habitants a moins de 14 ans. Le taux de chômage chez les $15-64$ ans s'élève à $38 \%$ avec un revenu fiscal de référence de 7270 euros en 2011. Soixante-cinq et demi pour cent $(65,5 \%)$ des habitants du quartier sont sans diplôme ou certificat d'études primaires (Soulet, 2014).

${ }^{15}$ Parmi les dix familles rencontrées, seuls deux pères étaient présents lors des entretiens.

${ }^{16}$ Ce type de carnet a déjà été utilisé dans d'autres enquêtes sur les mobilités urbaines des adolescents (Ramadier, Petropoulou, \& Bronner, 2008).
}

vacances scolaires, les informations relatives aux activités physiques pratiquées. La récupération de ces carnets auprès des enfants a été l'occasion d'un deuxième entretien plus court consistant à compléter ou préciser certains éléments, toujours en présence d'au moins un des parents.

L'ensemble des données recueillies permet non seulement de connaître les différentes modalités de pratique physique quotidienne (sport, marche/vélo, jeux physiques...) et le rapport aux activités physiques des enfants et de leurs parents, mais également leurs connaissances et représentations relatives aux recommandations de santé publique en la matière. Associées à la division sexuée des rôles parentaux, les trajectoires sportives et sociales des parents révèlent une pluralité de logiques familiales dans la transmission du goût à l'activité physique.

\section{Une diversité de rapports à la santé, à l'activité physique et au surpoids}

Concernant les recommandations de santé en matière d'alimentation, d'activité physique ou de santé en général, chaque enfant était invité à distinguer, au sein d'une liste préétablie ${ }^{17}$; les cinq comportements les plus favorables à la santé et les cinq comportements les plus défavorables. Le parent était ensuite convié à donner son sentiment sur les choix effectués par son enfant. Les résultats indiquent des différences entre les enfants participant au dispositif PRECCOSS et ceux n'y participant pas. Les premiers

\footnotetext{
${ }^{17}$ Exemples d'affirmations proposées au choix: «manger au moins 5 fruits et légumes par jour», «grignoter devant la télévision », «regarder du sport à la télé », «prendre les escaliers plutôt que l'ascenseur», «faire du sport tous les jours ».
} 
citent spontanément les messages sanitaires diffusés notamment dans les bandeaux d'informations ${ }^{18}$ accompagnant les publicités à la télévision. Ils repèrent davantage ceux en rapport avec l'alimentation, même s'ils n'oublient pas l'activité physique avec des affirmations comme «jouer au ballon» ou «bouger dans la cour de récréation». Les recommandations de santé sont également abordées dans le cadre des actions développées au sein du dispositif PRECCOSS : «le samedi le matin il y avait sport comme d'habitude et l'après-midi y avait une activité et on a parlé de la santé, des goûters et de tout» (N.S., fille, 9 ans, PRECCOSS). Des désaccords apparaissent parfois entre parent et enfant qui renvoient à des conceptions de la santé plus ou moins larges. Cela transparaît, par exemple, dans cet échange entre une mère et sa fille au sujet de l'affirmation «aller voir un spectacle» considérée par la fille comme une activité sédentaire donc «défavorable»à la santé. À l'inverse, la mère défendra l'aspect «favorable» à la santé en indiquant: «parce que tu sors tu vois du monde et ça fait du bien!» (mère de L.L., fille, 10 ans, PRECCOSS). On voit ici une pluralité de définitions de l'activité physique favorable à la santé : à la différence de sa fille, la mère considère l'activité physique au sens le plus large.

Les enfants ne participant pas au dispositif PRECCOSS montrent plus d'hésitations quant à leurs choix et ne sélectionnent pas les messages d'informations sanitaires évoqués plus haut. Parfois, les choix opérés et les justifications proposées paraissent même en contradiction avec les recommandations générales de santé. Par exemple, une fille a déclaré qu'il valait mieux ne pas prendre de petit déjeuner le matin: «prendre un petit déjeuner tous les matins. Ça je ne le fais pas. Parce que à l'école il y a des trucs, des quelques choses qu'on nous donne». Ce que confirme également sa mère : «c'est pour ça la plupart du temps elle évite à manger à la maison le matin. Parce qu'à l'école y a les fruits, y a des fois les légumes qui donnent tout le temps aux enfants. Elle m'a dit: Maman, moi je mange pas à la maison jusqu'à midi ». Le petit déjeuner est ainsi abandonné au profit d'une collation en cours de matinée alors même qu'il est présenté $^{19}$ comme un repas très important de la journée. La perception et l'interprétation de certaines actions peuvent conduire à l'adoption de pratiques éloignées des recommandations de santé publique.

\footnotetext{
${ }^{18}$ Un arrêté du 27/02/2007 fixe les conditions relatives aux informations à caractère sanitaire devant accompagner les messages publicitaires ou promotionnels en faveur de certains aliments et boissons. Ainsi, les publicités de ce type doivent être accompagnées de messages, comme par exemple «Pour votre santé, mangez au moins cinq fruits et légumes par jour», «Pour votre santé, pratiquez une activité physique régulière», «Pour votre santé, évitez de manger trop gras, trop sucré, trop salé». ${ }^{19}$ D'après les conseils préconisés sur le site Internet http: / www. mangerbouger.fr/ développé dans le cadre du Plan national nutrition santé.
}

De manière générale, l'analyse des entretiens fait ressortir trois rapports principaux aux activités physiques en fonction de la perception de leurs liens à la santé ${ }^{20}$.

\subsection{Un rapport « éloigné » à l'activité physique}

Trois familles ne participant pas au dispositif PRECCOSS attestent d'un rapport «éloigné» à l'activité physique tant en termes de pratiques proprement dites que de mise en relation avec d'éventuels effets sanitaires. L'importance de «manger mieux» et «bouger plus» est rappelée sans être reliée à des problématiques de santé. Ce rapport éloigné explique sans doute, en partie, le fait que les parents n'aient pas donné de suite à l'ordonnance médicale incitant leur enfant à rejoindre les activités du dispositif PRECCOSS.

\subsection{Un rapport « utilitaire » à l'activité physique}

Considérant principalement l'activité physique comme un outil de lutte contre la surcharge pondérale, deux autres familles présentent un rapport plutôt «utilitaire» comme les discours de certaines mères évoquant leur propre passé sportif pendant l'enfance en témoignent. Cette mère, par exemple déclare: «moi j'ai pas besoin parce que je suis maigre. Mais pour elle oui» (mère de C.M., fille, 8 ans, NON PRECCOSS). Une autre, en évoquant son enfance, justifie le fait de ne pas avoir pratiqué d'activité sportive de la sorte: «non moi j'étais pas en surpoids. Elle, à partir de trois ans, déjà elle a commencé à prendre, à prendre » (mère de M.D., fille, 10 ans, PRECCOSS). L'activité physique est conçue comme un instrument de prévention secondaire, ici de lutte contre le surpoids.

\subsection{Un rapport de « bien-être global » à l'activité physique}

Quatre familles enfin font preuve d'un rapport de «bien-être global» à l'activité physique. C'est le cas de cette mère qui découvre l'activité sportive en suivant des cours de fitness après quarante ans et développe une pratique plus intensive à la suite de la séparation d'avec son conjoint. Elle estime que cette activité « ça fait du bien mine de rien ». Elle se sent «plus forte, plus d'entrain quoi, ça fait du bien au corps. On sculpte un peu, sportive fine à nouveau». L'expérience personnelle des effets de la pratique l'incite à encourager son fils à pratiquer à son tour: «moi je vous dis : j'ai jamais été une grande sportive mais maintenant voilà avec l'âge et voilà, je m'y mets aussi. Et eux, ils sont jeunes, ils ont le dynamisme, ils ont tout. S'ils font rien, ils seront en mauvaise forme à 18 ans »

\footnotetext{
${ }^{20}$ Ce lien a déjà été questionné auprès d'adultes réalisant un bilan de santé et quatre profils avaient été mis en évidence: «physiquement inactif, le sentiment de ne pas être en bonne santé», «physiquement actif, orienté vers le plaisir et les loisirs », «physiquement actif au travail sans attention pour la santé » et «physiquement actif pour lutter contre le stress » (Perrin, Ferron, Gueguen, \& Deschamps, 2002).
} 
Tableau 2. Synthèse des habitudes de vie et des activités physiques des enfants enquêtés et participant à PRECCOSS.

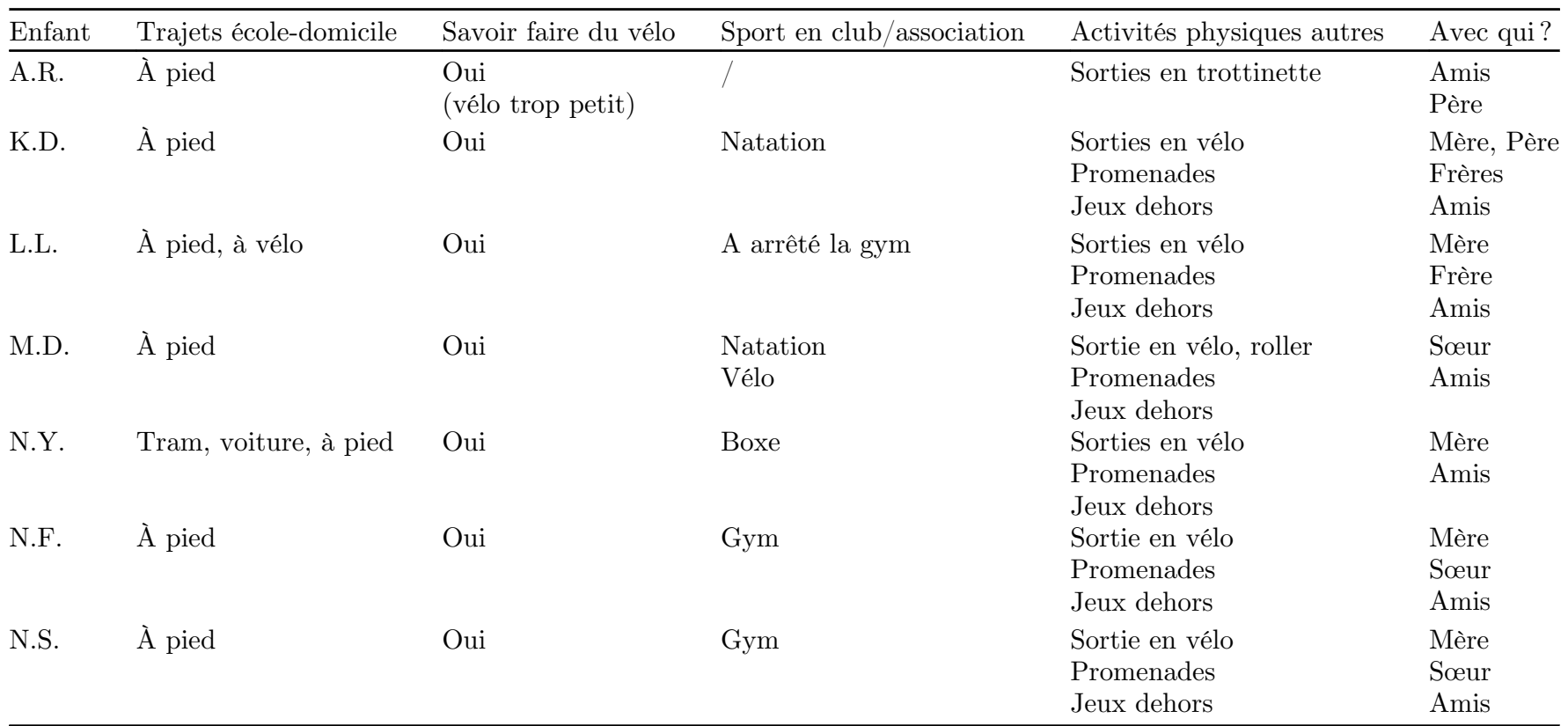

(mère de N.Y., garçon, 11 ans, PRECCOSS). Elle souhaite que ce dernier fasse le choix d'une activité sportive qui lui plaise pour favoriser une pratique régulière et continue. L'ensemble de ces familles a un enfant participant au dispositif PRECCOSS. L'action publique semble ici produire son effet dans la mesure où elle contribue à diffuser des représentations spécifiques des bienfaits de la pratique d'activités physiques (Berlivet, 2004). Il s'agira à présent, d'interroger l'impact des trajectoires et de l'histoire familiale dans la transmission d'un goût à l'activité physique.

\section{L'influence des trajectoires et de l'histoire familiale}

À la lumière des entretiens, plusieurs logiques familiales différenciées de transmission d'un goût à l'activité physique se dégagent. D'autre part, les habitudes de vie « actives » et l'activité physique semblent varier en fonction du passé migratoire des familles, des différences générationnelles mais également du lieu de vie de la famille.

\subsection{Logique familiale d'incitation voire d'obligation à l'activité physique}

Certains parents, eux-mêmes, pratiquants de sports tels que : l'aquagym, le fitness, la musculation, la course à pied ou encore le vélo, ont tendance à davantage inciter leur enfant à pratiquer une activité physique en club ou en association. Au-delà de la seule activité sportive encadrée, c'est surtout l'adoption par les parents d'habitudes de vie «actives » (déplacements à pied ou en vélo, fréquentation de parcs, sorties à la piscine, etc.) qui encourage la pratique physique des enfants eux-mêmes. Les familles dont l'enfant participe au dispositif PRECCOSS incitent davantage voire «obligent» leur enfant à pratiquer une ou des activités physiques ou sportives. Quatre enfants pratiquent ainsi en club, au minimum une fois par semaine, en plus de leur participation au dispositif. Ainsi en est-il de cette mère dont le fils voulait arrêter le basket: «je lui avais dit que je l'enlève pas du basket tant qu'il ne fera pas un autre sport» (mère de N.Y., garçon, 11 ans, PRECCOSS). De plus, ces enfants se rendent à l'école à pied ou à vélo ( $c f$. Tab. 2) accompagnés généralement de leur mère.

\subsection{Logique familiale de « libre choix » de l'activité physique}

Les enfants ne participant pas au dispositif PRECCOSS se caractérisent par l'absence de pratique sportive encadrée en club, tout comme leurs parents. Si certains parents affirment y avoir incité leur enfant, ils ne l'ont jamais imposée et ont laissé l'enfant libre de choisir de pratiquer ou non, comme cette mère qui explique «au foot aussi je l'ai inscrit, il va aller deux fois » puis abandonnera du fait de l'absence de ses copains du quartier (mère de $\mathrm{S}$. M., garçon, 9 ans, NON PRECCOSS). Cependant, les mères rencontrées expriment généralement le souhait de voir bouger plus leur enfant affirmant par exemple «non j'aime bien qu'elle fasse des activités vraiment! Parce que elle-même elle a envie, elle aime sortir, faire des activités » (mère de R.F., fille, 9 ans, NON PRECCOSS). Elles encouragent alors leur enfant à aller jouer dans un parc. Certains parents, surtout les pères, accompagnent aussi leur enfant lors de balades à vélo ou à la piscine. Toutefois, ces activités s'avèrent plus ponctuelles que régulières. Au quotidien par exemple, ces enfants vont plus souvent à l'école en voiture ( $c f$. Tab. 3) plutôt qu'à pied ou en vélo, dans un souci de gain de temps ou de moindre fatigabilité. 
Tableau 3. Synthèse des habitudes de vie et des activités physiques des enfants enquêtés et ne participant pas à PRECCOSS.

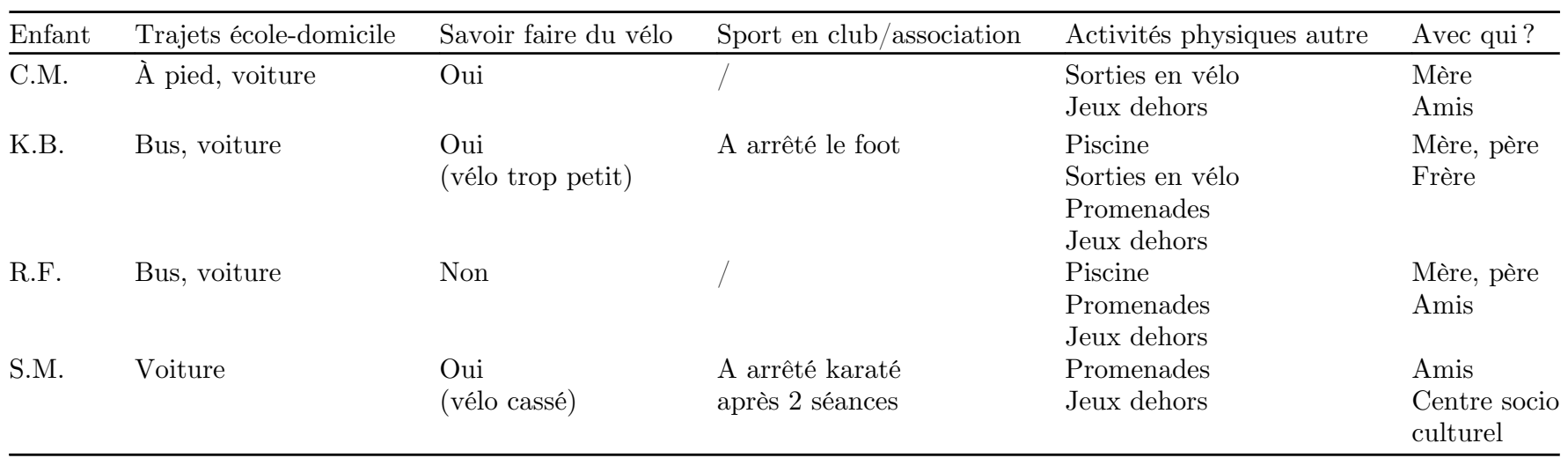

Une mère précise ainsi qu'elle «préfère la voiture parce que [sa fille] est très fatiguée. Ça fait la troisième année qu'on fait en bus. (...) Maintenant elle est fatiguée elle préfère... parce que en bus elle doit se réveiller tôt, elle doit se préparer avant pour pouvoir être à l'heure» (mère de R.F., fille, 9 ans, NON PRECCOSS).

\subsection{Un effet du parcours migratoire des familles}

Le passé migratoire des familles peut également expliquer leur faible engagement dans un style de vie actif. De ce fait, les parents de l'étude sont souvent moins enclins à inciter leurs enfants à pratiquer une activité physique. En effet, la plupart des parents rencontrés sont nés hors de France (Maroc, Algérie, Turquie, Russie, Congo ou Roumanie), leurs enfants étant quant à eux nés en France. Les parents des deux autres familles sont des immigrés de deuxième génération, les grands-parents étant nés en Turquie. Les effets de la «culture d'origine» des parents peuvent notamment s'apprécier par l'expression d'un rapport au corps (Boltanski, 1971) spécifique. Toutes les familles originaires d'Afrique et de Roumanie parlent d'une pratique sportive différente de celle qu'ils ont pu observer en France. En effet, pendant leur enfance, la seule expérience de pratique sportive «encadrée»se déroulait en milieu scolaire. Le sport «c'est juste avec l'école. Oui peut-être ils choisissent ceux qui ont plus envie et ils ajoutent une demi-journée dans la semaine pour faire du gymnase» (mère de R.F., fille, 9 ans, NON PRECCOSS, originaire du Maroc). Une autre mère originaire du Maroc explique que dans son enfance les activités physiques se pratiquaient à l'extérieur : «bon pour Maroc les activités comme ici c'est... y a pas. Ça n'existe pas. Oui, la vérité y a pas. Si tu veux faire des activités tu fais toimême. Oui! Toi-même, oui! C'est ça! Y a pas des centres, des trucs comme ça. Si tu veux faire des activités, tu fais toi-même. On joue cache-cache, (...) Ballon aussi! La balançoire! Oui, la corde à sauter. Oui, le (parle en arabe) je sais pas comment on dit. La marelle?! Oui on joue ça! Des trucs qu'on fait nous-mêmes. Alors ça oui. On trouve toujours quelque chose à faire. (...) On inventait carrément» (mère de N.S et N.F., filles, 9 et 10 ans, PRECCOSS, originaire du Maroc).

\subsection{Des différences générationnelles dans le mode de vie}

D'autres parents insistent également sur leur enfance plus «active» que celle de leurs enfants actuellement du fait de conditions d'existence différentes, mais aussi d'un accès facilité aujourd'hui à des loisirs plus sédentaires. «Oui je pense qu'à l'époque on était plus actif car il y avait pas des gadgets, des internet, tout ça. Maintenant non, ça prend beaucoup de temps. C'est ça qui l'intéresse pas des activités c'est dommage» (mère de K.D., garçon, 9 ans, PRECCOSS). Une mère originaire de Turquie soutient le même raisonnement: «oui quand j'étais petite, enfin nous étions beaucoup dehors. C'est plus comme maintenant avec les téléphones, les tablettes. Non, on faisait beaucoup de vélo, rollers, des trucs comme ça. On restait pas enfermé dans la maison!» (mère de L.L., fille, 10 ans, PRECCOSS).

\subsection{L'influence du lieu de vie sur l'activité physique}

La critique des jeux sédentaires masque toutefois les difficultés qu'éprouvent certains parents à autoriser leurs enfants à jouer librement dans leur quartier d'habitation. Beaucoup de parents se plaignent d'un manque de sécurité au sein de leur quartier avec notamment une circulation automobile ou des deux-roues jugées inadaptées et dangereuses. Ils ne souhaitent pas que leurs enfants traversent des routes tous seuls ou ne sortent sans être accompagnés. Le père de N.S. et N.F. (filles, 9 et 10 ans, PRECCOSS) livre son agacement et ses craintes pour ses enfants : "Le problème qu'il y a c'est le quartier, il est pas aussi sûr. Les scooters ils roulent sur le trottoir, les voitures qui roulent au lieu de 30, ils roulent à 100 donc c'est très vite dangereux, c'est très dangereux. Donc c'est pour ça, on leur donne un petit secteur qu'ils évitent au moins de traverser les routes quoi. [...] Franchement, faut être débile pour mettre un parc pour les enfants entre les routes. [...] Donc s'ils jouent au foot et y a un gamin qui jette la balle sur la route, y va y aller la chercher donc il va pas regarder. [...] C'est ça le problème, on peut pas laisser les enfants sortir comme ça». La perception du quartier et des possibilités d'indépendance des enfants varient aussi 
d'un contexte familial à l'autre, comme en atteste l'exemple de cette mère qui laisse son fils âgé de neuf ans sortir avec ses amis sans être surveillé par un membre de la famille (S.M., garçon, 9 ans, NON PRECCOSS). Il se rend également seul au centre socio-culturel où il joue surtout au football avec d'autres garçons du quartier.

Parmi les familles rencontrées, seules celles dont l'enfant ne participe pas au dispositif PRECCOSS se rendent au moins une fois par an dans le pays d'origine des parents souvent pour un mois au minimum. Dans le cadre de ces séjours, les enfants sont davantage autorisés à jouer à l'extérieur voire pour certains à se baigner ou à pratiquer des activités de loisirs aquatiques : «parce que voilà là-bas c'est autre chose et ben on a la mer, le soleil, le jet ski machin. B. là-bas, justement là-bas il fait beaucoup plus d'activités » (mère de K.B., garçon, 10 ans, NON PRECCOSS). Tous se disent beaucoup plus actifs dans leur pays d'origine. Les parents d'une famille expliquent «qu'au pays» ils se déplacent toujours à pied pour rendre visite à des membres de leur famille, alors que pour des distances identiques en France ils auraient pris la voiture ou les transports en commun (mère et père de N.S et N.F, filles, 9 et 10 ans, PRECCOSS). Ils ne craignent plus pour la sécurité de leurs enfants lorsqu'ils jouent sans surveillance parentale à l'extérieur.

Finalement, les habitudes de vie des parents marquées par leurs histoires personnelles et collectives permettent de comprendre la manière dont ils peuvent inciter leur enfant à pratiquer des activités physiques, voire à les faire participer au dispositif PRECCOSS, et à leur transmettre un «goût» pour ce type d'activités. Plus les parents sont eux-mêmes «actifs » et proposent des activités physiques, plus l'enfant sera actif à son tour. De plus, nos résultats semblent indiquer que l'expérience migratoire produit des effets sur le rapport des familles à l'activité physique. Ils nous invitent dès lors à tenir compte des relations entre «société de départ » et «société d'accueil » (Sayad, 1999). Par la suite, nous avons souhaité considérer l'influence parentale dans le cadre d'une division sexuée des rôles parentaux, en nous penchant plus spécifiquement sur les mères de familles qui étaient toujours présentes lors des entretiens.

\section{Une division sexuée des rôles parentaux}

Parmi les dix familles enquêtées, seules trois mères pratiquent une activité physique régulière. Deux d'entre elles «imposent » aussi à leur enfant la pratique d'un sport en club, comme cette mère qui veille à ce que sa fille ait une activité physique régulière, «toujours un sport au moins, une fois tous les deux jours quoi. Ça on essaye de faire. Souvent elles (en parlant de ses deux filles aînées) sortent faire un peu de vélo quand elle n'a pas le sport soit à l'école ou dans le club» (mère de M.D., fille, 10 ans, PRECCOSS). Une autre mère souligne également le caractère «obligatoire» de l'activité physique «bien sûr, je lui laisse pas le choix. Il est obligé de faire du sport» (mère de N.Y., garçon, 11 ans, PRECCOSS). Les pères, quant à eux, ne pratiquent pas davantage, ce qui peut s'expliquer, pour certains, par une dépense physique dans leur activité professionnelle, comme pour ce père qui ne fait pas de sport car «il travaille comme carreleur, il a beaucoup des activités pendant le travail» (mère de K.D., garçon, 9 ans, PRECCOSS). Nous observons un seul cas de figure où l'enfant a pratiqué la même activité sportive que son père. M.D., la fille ainée de la famille, a testé le karaté (sport passion du père) pendant un an, avant de choisir la natation. $\mathrm{Du}$ fait du nombre limité de familles et contrairement à d'autres enquêtes (Mennesson, 2011), il nous est difficile de montrer que le père transmettrait éventuellement sa «passion sportive».

Pour PRECCOSS aussi, ce sont les mères qui accompagnent généralement leur enfant aux activités, même les week-ends où le père bénéficie de son droit de garde (dans les cas de séparation des parents), «samedi je l'ai amené au PRECCOSS et on est allé à la boxe et le soir, il est allé chez son père» (mère de N.Y., garçon, 11 ans, PRECCOSS). Les pères apparaissent moins investis dans la prise en charge de l'activité physique de leurs enfants.

Pendant les périodes de vacances scolaires, le constat semble identique avec un accompagnement plus fréquent des mères notamment pour les activités d'extérieur (fréquentation de parcs et balades en vélo surtout). Par exemple, lors des vacances scolaires N.F. (fille, 10 ans, PRECCOSS) a joué dans un parc quasiment tous les jours, accompagnée par sa mère et d'autres membres de sa famille (tante, cousine). Avec son père, elle a partagé davantage d'activités sédentaires (comme regarder la télévision) ( $c f$. Fig. 2).

Les mères semblent plus présentes dans l'accompagnement au quotidien des activités physiques de leur enfant. L'importance qu'elles accordent à ces activités, leurs propres rapports aux éventuels bienfaits sanitaires de telles pratiques et leurs degrés d'incitation à ce type d'activités expliquent une part des habitudes de vie plus ou moins «actives» qu'elles transmettent à leur enfant. Certaines mères favorisent ces activités même si la participation au dispositif PRECCOSS peut dans certains cas être perçue comme une contrainte supplémentaire, comme l'explique cette mère à son fils : «Et après, faut aussi avoir le temps de pouvoir t'amener aussi chaque fois » (mère de N.Y., garçon, 11 ans, PRECCOSS).

Les mères jouent un rôle important dans la promotion de l'activité physique et l'incitation à la pratique. Elles semblent à l'origine de la décision d'inscrire ou non leur enfant aux activités du dispositif PRECCOSS. Leur compréhension $\mathrm{du}$ fonctionnement $\mathrm{du}$ dispositif semble essentielle. Une des mères rencontrées confond par exemple les activités proposées dans le cadre périscolaire avec celles du dispositif PRECCOSS, refusant ainsi d'y inscrire sa fille: «on a parlé mais on n'a pas accepté. $M$. (...) m'a dit que c'est très difficile après l'école tout de suite. Directement à faire PRECCOSS scolaire à faire le sport et tout ça. À 15 h45 elle sort de l'école, rester jusqu'à 17 h30 jusqu'à 18 h pour faire le PRECCOSS, elle m'a dit ça fait beaucoup » (mère de C.M., fille, 8 ans, NON PRECCOSS). Les mères les plus physiquement «actives » sont celles qui encouragent ou imposent davantage à leur 


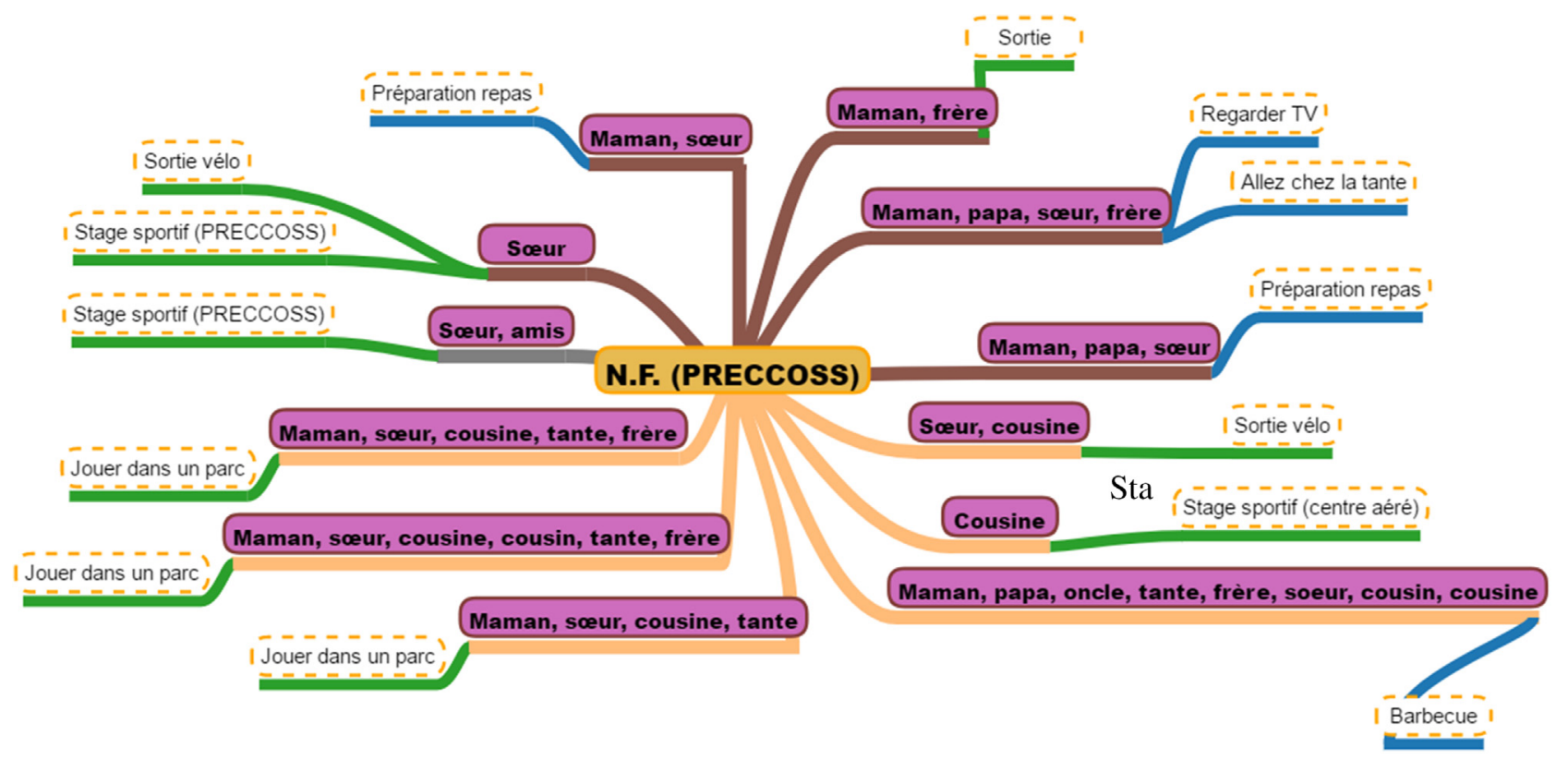

Fig. 2. Synthèse des activités de loisirs de N.F. hors jours scolaires.

enfant en surcharge pondérale la pratique d'activités physiques. La participation au dispositif PRECCOSS constitue alors une des activités possibles dans un ensemble plus large de pratiques physiques.

\section{Discussion}

L'un des objectifs de l'étude était de mieux comprendre le rapport des enfants et de leurs parents à la santé, au surpoids et aux recommandations de santé publique concernant la pratique d'activité physique. Il s'agissait également d'identifier des logiques familiales différenciées en fonction de différents facteurs : histoire sociale, migratoire et sportive des parents, lieu de vie de la famille, existence d'une division sexuée des rôles parentaux. Les résultats mettent en évidence une diversité de rapports à la santé, à l'activité physique et au surpoids dans le discours des enfants et de leurs parents. Deux logiques familiales principales ont été dégagées : une logique d'incitation (voire d'obligation) à l'activité physique et une logique de «libre choix». Les trajectoires et l'histoire familiale semblent influencer les habitudes de vie «actives» et le rapport à l'activité physique. L'étude souligne un décalage entre le modèle corporel dominant véhiculé par les politiques de santé publique et la culture corporelle de ces familles mis en évidence par d'autres travaux (Martin-Criado, 2015; Peretti-Watel, 2000; Régnier \& Masullo, 2009). La dernière partie des résultats révèle enfin l'effet de la division sexuée des rôles parentaux sur le rapport des enfants à l'activité physique de santé.

Au-delà des représentations et des connaissances relatives à la santé, c'est également la prise en compte de la surcharge pondérale comme «souci» de santé qu'il convient de questionner. En effet, plusieurs études
(Carnell et al., 2005; Mutzig, 2017; Tanas, Pedretti, Gilli, Gagnayre, \& Marcolongo, 2011) ont démontré que les parents n'ont pas toujours conscience du surpoids ou de l'obésité de leur enfant et de leurs éventuelles conséquences en termes de santé, ne les incitant pas de fait à des actions particulières ou à une prise en charge spécifique. En cours d'entretien, les parents interrogés ayant leur enfant inscrit aux activités du dispositif PRECCOSS abordent spontanément la situation de surpoids ou d'obésité de leur enfant. Même s'ils n'évoquent jamais de potentiels risques sur la santé dans les années à venir, ils expriment leur souhait d'agir pour rétablir un poids «normal» chez leur enfant. Les parents enquêtés dont l'enfant ne participe pas au dispositif PRECCOSS affirment moins la nécessité de modifier leurs habitudes de vie pour lutter contre la surcharge pondérale de leur enfant.

Comme d'autres travaux sur les quartiers populaires l'ont montré (Aceti \& Vieille Marchiset, 2014; VieilleMarchiset et al., 2014), les connaissances des enfants et des parents relatives aux recommandations de santé renvoient principalement aux aspects nutritionnels et peu ou moins à l'activité physique. Il est ainsi notable qu'aucune des familles rencontrées n'ait $\mathrm{pu}$ indiquer précisément le nombre de pas recommandé pour être considéré comme une personne «active» ${ }^{21}$. La connaissance des recommandations en termes d'activités physiques reste assez floue. Les parents se réfèrent principalement à l'injonction «bouger» sans donner d'indications de fréquence, de durée ou d'intensité de pratique. L'analyse des entretiens fait ressortir trois rapports principaux aux activités

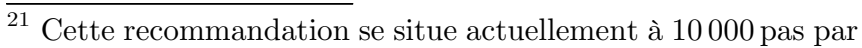
jour.
} 
physiques en fonction de la perception de leurs liens à la santé: un rapport «éloigné», «utilitaire» ou «de bienêtre global».

Finalement, la participation au dispositif PRECCOSS semble fortement distinguer les familles du point de vue de leurs rapports aux activités physiques dans une perspective sanitaire. En cas de participation, les mères cherchent à réguler le poids de leur enfant dans un but de lutte contre la surcharge pondérale. Les parents comme les enfants connaissent alors les recommandations de santé et en parlent ensemble. Pour les familles dont l'enfant ne participe pas au dispositif, la prise de conscience du surpoids ou de l'obésité n'est pas aussi marquée et les recommandations de santé ne semblent pas toujours comprises.

Les rapports des parents aux activités physiques dans leur lien à la santé varient et sont susceptibles d'expliquer des degrés différenciés d'incitation des enfants à la pratique d'activités physiques au quotidien. L'encouragement à la pratique d'activités sportives semble pouvoir être mis en lien avec l'histoire sportive des parents, mais aussi avec leur histoire sociale de manière plus large tenant compte notamment de leurs expériences de la migration et de leur nouvel environnement de vie. En fonction du parcours personnel de chacun des membres de la famille (Seraphin, 2011), des trajectoires sociales et de l'histoire familiale «faite corps » (Bourdieu, 1980, 1997), nous avons pu identifier deux logiques familiales différenciées.

De plus, nos résultats corroborent davantage l'idée selon laquelle le «goût» pour l'activité physique au sens large serait transmis principalement par les mères (Mennesson, 2011). Elles semblent jouer un rôle déterminant dans la mesure où elles imposent plus fréquemment un mode de vie actif aux enfants. Elles encouragent la constitution et le maintien, chez leur enfant, d'habitudes de pratique d'activité physique et elles participent également à développer une perception positive de ses compétences sportives (Bois, 2003; Bois \& Sarrazin, 2006). Plus les mères sont investies dans une activité physique, plus leurs enfants (fille ou garçon) pratiqueraient (Bois, 2003). Ces résultats mériteraient évidemment des approfondissements en tenant compte de l'origine sociale des mères (Martin-Criado, 2015). Au quotidien, ce sont majoritairement elles qui s'occupent d'amener les enfants à l'école et à leurs activités. Elles se chargent également du suivi du travail scolaire, du travail domestique de santé (Cresson, 1995) et des éventuels rendez-vous médicaux.

Les mères sont de plus les premières interlocutrices du médecin prescripteur de cette participation au dispositif. Certaines mères la jugent probablement légitime car relevant d'injonctions sanitaires et cherchent à y répondre sur le modèle de la «bonne mère » (Martin-Criado, 2015). Toutefois, dans les milieux populaires des tensions fréquentes existent entre le respect de ces injonctions et une forme de liberté par rapport aux normes de prévention et de corpulence (Martin-Criado, 2015; Régnier \& Masullo, 2009; Thin, 1997, 2005). Les familles ne participant pas à PRECCOSS ne se conforment pas aux injonctions dont elles sont destinataires. Souvent plus en difficultés avec l'usage et la compréhension de la langue française, elles s'avèrent aussi moins «actives» et promptes à considérer la surcharge pondérale comme un souci de santé.

\section{Conclusion}

L'analyse des parcours de prévention par les activités physique et/ou sportives d'enfants en surpoids ou obèses dans un quartier populaire à Strasbourg met en évidence le poids des transmissions familiales et plus particulièrement le rôle important joué par certaines mères. Au-delà d'une forme affirmée d'inculcation, elles accompagnent aussi régulièrement leurs enfants dans le cadre de leurs activités physiques et sportives (déplacements actifs pour se rendre à l'école, inscription de l'enfant dans un club sportif, accompagnement régulier dans des parcs de la ville et aux activités sportives du dispositif PRECCOSS). La transmission d'un «goût» pour l'activité physique semble davantage s'opérer ici par imprégnation (Octobre \& Jauneau, 2008).

Les enfants participant au dispositif PRECCOSS sont plus actifs au quotidien et pratiquent - pour la plupartune activité sportive en club. Leurs parents ont des habitudes de vie plus «actives» que les parents dont l'enfant ne participe pas au dispositif, et ils perçoivent l'activité physique comme propice à un «bien-être global ». Ils se conforment davantage aux recommandations de santé publique. Il est toutefois difficile d'affirmer que ces parents et leurs enfants ont transformé leurs pratiques dans le sens d'une autocontrainte plus grande en intégrant volontairement dans leur quotidien et mode de vie les préconisations sanitaires en termes de pratique d'activité physique. En effet, dans les familles populaires les pratiques sont davantage liées à des contraintes extérieures (comme ici la prescription médicale) plutôt qu'elles ne visent à générer une autocontrainte chez leurs enfants (Thin, 2005). La question de la durabilité et de l'intensité des pratiques se pose alors. L'activité physique des enfants pourrait d'ailleurs faire l'objet, dans de prochaines études, d'évaluations plus précises - en termes de quantité, de régularité et d'intensité - avec l'utilisation d'instruments de mesure spécifiques comme par exemple des accéléromètres, des GPS $^{22}$ voire des applications dédiées pour smartphone.

Nos résultats montrent l'importance d'étudier les processus de socialisation en tenant compte des trajectoires individuelles et sociales des membres de la famille (Blöss, 1996). Mais les recommandations des médecins et professionnels de santé s'inscrivent également dans un système de normes et modèles d'esthétique corporelle définissant le corps enfantin légitime qui influence plus ou moins les parents. Le problème public de l'obésité et du surpoids renvoie en effet à la place accordée à l'individu dans la société et à une définition morale tant de l'ordre

\footnotetext{
${ }^{22}$ Voir par exemple les méthodes des géographes pour mesurer les mobilités urbaines (Misslin et al., 2015).
} 
social que du «bon» poids ou de la «bonne» apparence physique. Or, au-delà d'un rapport uniforme des parents au surpoids, l'enquête indique que l'adhésion à une forme de gouvernement des corps (Fassin \& Memmi, 2004) est facilitée pour les parents - et surtout les mères - convaincus, parfois pour l'avoir expérimenté eux-mêmes, des bienfaits de l'activité physique et de la nécessité d'agir face à la situation de surcharge pondérale de leur enfant. Ce processus de conformation a sans doute un effet d'intériorisation marquant les biographies (Chantegros, Orange, Pegourdie, \& Rougier, 2012). Des formes de résistance aux injonctions normatives sont également perceptibles et renvoient probablement aux rapports de défiance qu'entretiennent certaines familles populaires avec les institutions (comme l'école par exemple) (Thin, 1998). Les parcours de prévention des enfants par le sport ne sont pas uniformes et dépendent fortement de la socialisation familiale à resituer elle-même dans la complexité des expériences socialisatrices (Lahire, 2013). Si nous avons pu souligner le rôle joué par les mères, il nous faudra encore questionner plus en détail les effets de la socialisation sur la différenciation sexuelle des rapports au corps (Court, 2010). Notre échantillon très réduit nous incite également à une grande prudence dans l'éventuelle généralisation des éléments mis en évidence qui devront être confirmés à plus large échelle.

En guise de perspective, s'attachant à étudier spécifiquement le rôle des parents, cette première enquête doit être complétée par l'analyse d'autres facteurs influençant le rapport à l'activité physique des enfants, notamment le rôle d'autres «acteurs » (grandsparents, frères, sours, oncles, tantes, professeurs des écoles, éducateurs, voisins, amis...). Ensuite, notre étude concernait uniquement des familles vivant dans un espace urbain où l'offre en activités physiques est beaucoup plus riche qu'en milieu rural où il faut se déplacer pour se rendre par exemple à la piscine. Le territoire influençant également le rapport à l'activité physique, il s'agira dans une étude ultérieure de comparer des populations d'enfants résidant dans deux territoires différents afin de vérifier si la distance géographique ne redouble pas la distance sociale et culturelle.

Une meilleure connaissance du rapport des bénéficiaires à cette action publique spécifique nous semble susceptible d'apporter aux acteurs locaux quelques éléments d'évaluation et d'aide à la décision. Prenant appui sur les résultats de l'étude, des préconisations peuvent ainsi être formulées à destination notamment des mères des quartiers populaires, sous la forme d'ateliers participatifs par exemple (d'activité physique notamment). L'un des objectifs serait, par ce biais, de diffuser les bonnes pratiques physiques par l'échange direct avec les populations concernées, en vue d'encourager la participation au dispositif PRECCOSS des enfants des familles les moins réceptives aux politiques publiques de prévention et les moins convaincues a priori par un tel engagement.

\section{Déclaration de contribution des auteurs}

Tous les auteurs ont également contribué à la production et à la rédaction de cet article.

\section{Thanks}

The authors thank the Regional Health Agency Grand Est, the city of Strasbourg and the General Commissariat for Territorial Equality for the funding granted to this research project within the framework of the city contract Eurometropole of Strasbourg (2015-2020).

\section{Author contribution statement}

All authors contributed equally to the production and writing of this article.

Remerciements. Les auteurs remercient l'Agence Régionale de Santé Grand Est, la ville de Strasbourg et le Commissariat général à l'égalité des territoires pour le financement accordé à ce projet de recherche dans le cadre du contrat de ville de l'Eurométropole de Strasbourg (2015-2020).

\section{Références}

Aceti, M., \& Vieille Marchiset, G. (2014). Un capital salutaire à transmettre. Analyse comparative des programmes de promotion de la santé par l'activité physique en Europe. JuriSport, 140, 41-45.

ANSES. (2016). Rapport d'expertise collective. Actualisation des repères du PNNS-Révisions des repères relatifs à l'activité physique et à la sédentarité.

Attias-Donfut, C., \& Rozenkier, A. (dir.). (1995). Les solidarités entre générations vieillesse, familles, État. Paris: Nathan.

Auger, F., Lefrançois, C., \& Trepied, V. (2017). Penser l'âge dans l'enquête et ses enjeux. Sociologies. Disponible sur http:// sociologies.revues.org/5990.

Berger, P., \& Luckmann, T. (1986). La construction sociale de la réalité. Paris: Armand Colin.

Berlivet, L. (2004). Une biopolitique de l'éducation pour la santé. La fabrique des campagnes de prévention. In D. Fassin \& D. Memmi (dir.), Le gouvernement des corps (pp. 37-75). Paris : EHESS.

Blöss, T. (1996). Éducation familiale et beau-parenté. L'empreinte des trajectoires biographiques. Paris: L'Harmattan.

Bois, J. (2003). Socialisation de l'activité physique et des perceptions de compétence sportive: Le rôle des parents chez l'enfant et le préadolescent. Thèse de Doctorat STAPS. Université Joseph Fourier, Grenoble 1.

Bois, J., \& Sarrazin, P. (2006). Les chiens font-ils des chats? Une revue de littérature sur le rôle des parents dans la socialisation de leur enfant pour le sport. Sciences et Motricité, 57(1), 9-54.

Boltanski, L. (1971). Les usages sociaux du corps. Annales. Économies, Sociétés, Civilisations, 1, 205-233.

Bourdieu, P. (1980). Le Sens practique. Paris: Minuit.

Bourdieu, P. (1997). Méditations pascaliennes. Paris : Seuil.

Carnell, S., Edwards, C., Croker, H., Boniface, D., \& Wardle, J. (2005). Parental perceptions of overweight in 3-5 years old. International Journal of Obesity, 29, 353-355.

Caspersen, C.J., Powell, K.E., \& Christenson, G.M. (1985). Physical activity, exercise, and physical fitness: Definitions and distinctions for health-related research. Public Health Reports (Washington, D.C. : 1974), 100(2), 126-131. 
Chantegros, S., Orange, S., Pegourdie, A., \& Rougier, C. (dir.). (2012). La fabrique biographique. Limoges: Presses Universitaires de Limoges.

Court, M. (2010). Corps de filles, corps de garçons: Une construction sociale. Paris: La Dispute.

Court, M., Mennesson, C., Salaméro, É., \& Zolesio, E. (2014). Habiller, nourrir, soigner son enfant : La fabrication de corps de classes. Recherches familiales, 11, 43-52.

Craggs, C., Corder, K., Van Sluijs, E., \& Griffin, S. (2011). Determinants of change in physical activity in children and adolescents. A systematic review. American Journal of Preventive Medicine, 6, 645-658.

Cresson, G. (1995). Le travail domestique de santé. Paris: L'Harmattan.

Darmon, M. (2007). La socialisation. Paris: Armand Colin.

Déchaux, J. (2009). Sociologie de la famille. Paris : La Découverte.

Defrance, J. (1995). Sociologie des Sports. Paris : La Découverte.

DREES (Direction de la recherche, des études, de l'évaluation et des statistiques) et Santé publique France. (2017). L'état de santé de la population en France. Rapport 2017. Disponible sur http://drees.solidarites-sante.gouv.fr/.

Fassin, D., \& Memmi, D. (dir.). (2004). Le gouvernement des corps. Paris : EHESS.

Faure, S. (2000). Apprendre par corps. Socio-anthropologie des techniques de danse. Paris: La Dispute.

Favier-Ambrosini, B. (2016). Socio-histoire du lien entre activité physique et santé de 1960 à 1980. Santé Publique, 28, 15-24.

Fernandez, D., Polesi, H., Schweitzer, B., Daniele, L., Schauder, N., Seiller, M., Kochanowski, J., \& Imbert, F. (2007). Inégalité sociale des enfants face au surpoids en Alsace: Données de la visite médicale d'admission en école élémentaire, France, 2001-2002. Bulletin Épidémiologique Hebdomadaire, 2-3, 20-23.

Gasparini, W., \& Knobé, S. (2013). La prescription médicale d'activité physique: Quels effets sur la santé des strasbourgeois? Analyse qualitative du rapport à la pratique des patients. Rapport de recherche pour l'Agence régionale de santé d'Alsace.

Gasparini, W., Knobé, S., \& Didierjean, R., (2015). Physical activity on medical prescription: A qualitative study of factors influencing take-up and adherence in chronically ill patients, Health Education Journal, 6, 720-731.

Guignon, N., Collet, M., Gonzalez, L., De Saint Pol, T., Guthmann, J-P., \& Fonteneau, L. (2010). La santé des enfants en grande section de maternelle en 2005-2006. Études et résultats, DREES, 737.

Haute Autorité De Santé. (2011). Surpoids et obésité de l'enfant et de l'adolescent. Synthèse des recommandations de bonne pratique. Disponible sur https://www.has-sante.fr/.

Lahire, B. (2013). Dans les plis singuliers du social. Paris: La Découverte.

Martin-Criado, E. (2015). L'ambivalence du contrôle du poids chez les mères de famille des classes populaires. Actes de la recherche en sciences sociales, 208, 74-87.

Mauss, M. (1950). «Les techniques du corps». In M. Mauss, Sociologie et anthropologie, Paris: PUF, pp. 365-386 (Communication présentée à la Société de Psychologie le 17 mai 1934).

Mennesson, C. (2011). Socialisation familiale et investissement des filles et des garçons dans les pratiques culturelles et sportives associatives. Réseaux, 168-169, 87-110.

Misslin, R., Charreire, H., Weber, C., Enaux, C., Bastian, T., Simon, C., \& Oppert, J-M. (2015). Mobilités actives et santé : Apports et limites d'un protocole de mesure de la marche et du vélo combinant des capteurs de mouvements (GPS et accéléromètres). Cybergeo : European Journal of Geography. Disponible sur http://journals.openedition.org/cybergeo/ 26711.

Mitchell, J., Skouteris, H., McCabe, M., Ricciardelli, L.A., Milgrom, J., Baur, L.A., \& Dwyer, G. (2012). Physical activity in young children: A systematic review of parental influences. Early Child Development and Care, 182(11), 14111437.

Mutzig, N. (2017). Parentalité et prise en charge de l'enfant en surpoids ou obèse. Étude qualitative auprès de 17 familles strasbourgeoises bénéficiaires du dispositif PRECCOSS. Thèse de médecine, Mention D.E.S. Santé Publique et Médecine Sociale. Université de Strasbourg.

Neyrand, G., Mennesson, C., \& Fine, A. (2014). Enfance, corps et genre. Sciences Humaines, 258. Disponible sur https://www. scienceshumaines.com/normenfanenfance-corps-et-gen re fr 32383.html.

O'Connō, T.M., Jago, R., \& Baranowski, T. (2009). Engaging parents to increase youth physical activity. A systematic review. American Journal of Preventive Medicine, 37(2), 141-149.

Octobre, S., \& Jauneau, Y. (2008). Tels parents, tels enfants? Une approche de la transmission culturelle. Revue française de sociologie, 4, 695-722.

Peretti-Watel, P. (2000). Sociologie du risque. Paris: Armand Colin.

Peretti-Watel, P., \& Moati, J-P. (2009). Le principe de prévention. Le culte de la santé et ses dérives. Paris: Seuil.

Perrin, C., Ferron, C., Gueguen, R., \& Deschamps, J-P. (2002). Lifestyle patterns concerning sports and physical activity, and perceptions of health. Sozial- und Präventivmedizin, 47, 162-171.

Pociello, C. (1999). Les cultures sportives. Paris: PUF.

Poulain, J.-P. (2009). Sociologie de l'obésité. Paris: PUF.

Radel, A. (2012). 50 ans de campagnes d'éducation pour la santé. L'exemple de la lutte contre la sédentarité et de la promotion des modes de vie actifs (1960-2010). Thèse de doctorat STAPS. Université de Toulouse.

Ramadier, T., Petropoulou, C., \& Bronner, A-C. (2008). Quelle mobilité quotidienne intra-urbaine sans la voiture? Le cas des adolescents d'une banlieue de Strasbourg. Enfance, famille et Génération, 8. Disponible sur http://www.erudit.org/revue/ efg $/ 2008 / \mathrm{v} / \mathrm{n} 8 /$ index.html.

Régnier, F., \& Masullo, A. (2009). Obésité, goûts et consommation. Intégration des normes d'alimentation et appartenance sociale. Revue française de sociologie, 4, 747-773.

Saint Pol (de), T. (2007). L'obésité en France: Les écarts entre catégories sociales s'accroissent. INSEE Première, 1123.

Saint Pol (de), T. (2008). Obésité et milieux sociaux en France: Les inégalités augmentent. Bulletin épidémiologique hebdomadaire, 20, 175-179.

Sayad, A. (1999). La double absence. Des illusions de l'émigré aux souffrances de l'immigré. Paris: Seuil.

Seraphin, G. (2011). Introduction: Lien intergénérationnel et transmissions. Recherches Familiales, 8, 3-6.

Signorini, E., Ringenbach, S., Mutzig, N., \& Alizada, U. (2015). Rapport d'activité 2015 PRECCOSS. Strasbourg: Service promotion de la santé de la personne.

Signorini, E., Colle, J., Mutel, T., Ringenbach, S., Liehn, G., Lix, C., \& Ulrich, V. (2016). Rapport d'activité 2016 PRECCOSS. Strasbourg: Service promotion de la santé de la personne.

Skouteris, H., McCabe, M., Ricciardelli, L.A., Milgrom, J., Baur, L.A., Aksan, N., \& Dell'Aquila, D. (2012). Parent-child interactions and obesity prevention: A systematic review of the literature. Early Child Development and Care, 2, 153-174. 
Soulet, B. (2014). Atlas thématique et recueil d'indicateurs sur les $Q P V$ de la CUS. Service géomatique et connaissance $d u$ territoire, Direction de l'Urbanisme, de l'Aménagement et de l'habitat.

Tanas, R., Pedretti, S., Gilli, G., Gagnayre, R., \& Marcolongo, R. (2011). Évaluation clinique d'un programme d'éducation thérapeutique centré sur les familles en surpoids. Éducation thérapeutique du patient/Therapeutic patient education, 3, 111-120.

Thin, D. (1997). "Tant qu'on a la santé...". Des familles populaires et de la santé de leurs enfants. Rapport d'étude sociologique. Disponible sur https://halshs.archives-ouver tes.fr/halshs-00475253.

Thin, D. (1998). Quartiers populaires. L'école et les familles. Lyon: Presses Universitaires de Lyon.

Thin, D. (2005). Les familles populaires sous l'emprise des logiques éducatives dominantes à travers la scolarisation. Carnets de bord, 10, 67-78.
Trost, S.G., McDonald, S., \& Cohen, A. (2013). Measurement of general and specific approaches to physical activity parenting: A systematic review. Childhood Obesity, 9(SUPPL. 1), S40-S50.

Vieille-Marchiset, G., Knobé, S., Didierjean, R., Aceti, M., Digennaro, S., \& Grimminger, E. (2014). Corps en mouvement, corps en santé. Regard socio anthropologique sur des enfants de quartiers pauvres en Europe. L'Observatoire, 80, 9-13.

Vincelet, C., Galli, J., \& Gremy, I. (2006). Surpoids et obésité en Ile-de-France. Observatoire Régional de Santé d'Tlle-deFrance. Disponible sur http://www.ors-idf.org/.

Wacquant, L. (2000). Corps et âme. Carnets ethnographiques d'un apprenti boxeur. Marseille: Agone.

Whitaker, R.C., Wright, J.A., Pepe, M.S., Seidel, K.D., \& Dietz W.H. (1997). Predicting obesity in young adulthood from childhood. The New England Journal of Medicine, 13, 869-873.

Citation de l'article : Grassler M, Knobé S, \& Gasparini W (2019) Les parcours de prévention par le sport de l'obésité chez les enfants d'un quartier populaire strasbourgeois : transmissions familiales et rapports à l'activité physique. Mov Sport Sci/Sci Mot, https://doi.org/10.1051/sm/2019002 